\title{
The Crack Evolution and Damage Development of Coal Uniaxial Compression Failure under Different Gas Pressure
}

\author{
HAN Peibo ${ }^{1, a}$ \\ 1. College of resource and environmental science, Chongqing University, Chongqing 400044, \\ China;a.hanpb@163.com
}

Key words:raw coal;gas pressure;uniaxial compression;micro-crack development;damage

Abstract: To study the influence of gas pressure to coal failure process and crack evolution, using coal solid-gas coupled mechanics testing system, conducted the raw coal uniaxial compression failure experiment under different gas pressure; the evolution process of surface micro-cracks was synchronously observed and recorded, and was studied in comparison with coal macroscopic failure process; then the damage variable D of coal sample was defined based on elasticity modulus, and the evolution of $\mathrm{D}$ through macroscopic failure process was studied. The results show: cracking tips on coal surface appear at the yield stage, then start to develop rapidly after peak stress, and the coalescence of main crack happens at post-peak stage; the bigger gas pressure is, the lower the peak stress is; the exist of adsorption gas can delay the onset of surface crack initiation; the damage variable $\mathrm{D}$ begin to increase at yield stage, rise sharply after peak stress, and finally reached nearly 1 when the coal sample failed.

\section{Introduction}

With the increasing depth and intensity of coal mining, coal and rock dynamic disasters such as underground impact pressure and coal and gas outburst occurred more frequently. The occurrence of coal and rock dynamic disasters associated with the destruction of the coal, To some extent, Mesoscopic fracture morphology on the surface of the coal can reflect the Damage form and structure characteristics during coal damage process. Therefore the macroscopic damage of coal can be studied by truly recording the Coal surface crack extension and evolution process.

At present, mesoscopic mechanics test for rock materials at home and abroad mainly through the following ways: SEM, CT, holographic interferometry and optical microscopy. However, Compared with the laboratory equipment of $\mathrm{SEM}^{[1] \sim[5]}, \mathrm{CT}^{[6] \sim[8]}$, and holographic interferometry ${ }^{[9] \sim}$ ${ }^{[10]}$, it is more economic and practical to use the test system constituted by Optical microscope and loading device to carry out Mesoscopic mechanics experiments ${ }^{[11] \sim[13]}$.

The combination of ground stress, gas and physical and mechanical properties of coal and rock is the main factor that induced coal and rock dynamic disasters. So the study of dynamic evolution process of coal damage affected by gas pressure variation is of great significance to coal mine safety production. In this paper, the growth and expansion process of coal crack under different gas pressure was analyzed based on the raw coal as the research object, and the damage evolution regularity during the coal failure is also researched.

\section{Experimental method}

\section{The test device and specimen preparation}

This experiment used Shimadzu AG-I $250 \mathrm{kN}$ electronic material testing machine as loading device, and "Coal and rock solid/gas coupling mechanics test device" ${ }^{[14]}$ as experimental cavity, including loading system, pneumatic system, airtight device, data acquisition and control system. 
The raw coal specimens were from F15\# coal seam in 10th coal mine of PingDingShan Coal Group. The raw coal sample were cored and cut into $\Phi 50 \mathrm{~mm}$ x $100 \mathrm{~mm}$ standard cylindrical specimens, and then put into the stoving oven for $24 \mathrm{~h}$. To avoid the end effect, polishing the top surface and bottom of coal specimen by $800 \mathrm{Cw}$ sandpaper, smearing Vaseline on the top and bottom, and placing an iron gasket between specimen and pressure head.

\section{The test process}

Firstly the test specimen is closed in solid-gas coupling mechanics test device, then filling different pressure gas into the device and adsorbing for $24 \mathrm{~h}$. Using Shimadzu AG-I 250kN electronic material testing machine as loading device to carry out the uniaxial loading on coal specimen through the obturator piston rod and record data; loading by displacement control and keep the rate constant; observe and record through camera throughout the glass window. In order to research the evolution process of coal crack development under different gas pressure, conduct the experiment respectively in $0 \mathrm{MPa}, 1 \mathrm{MPa}$ and $2 \mathrm{MPa}$ gas pressure condition, and record the real-time dynamic observation by camera.

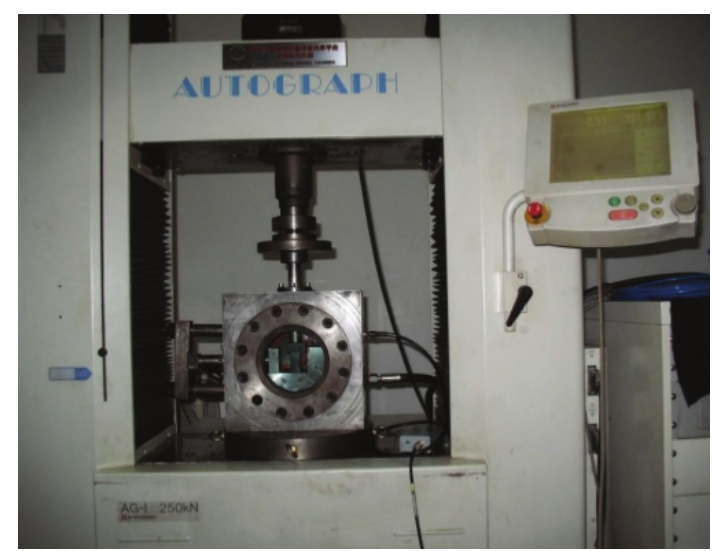

Fig.1 Testing apparatus of coal solid-gas coupled mechanics

\section{The test results}

\section{Coal sample crack extension development}

Based on the data record and real-time video of coal specimen damage process, select key points in its $\sigma-\varepsilon$ curve as the observation point, and find out the observation pictures of corresponding moment from the video, thus obtain analysis of coal sample surface crack expansion process under different gas pressure as follows.

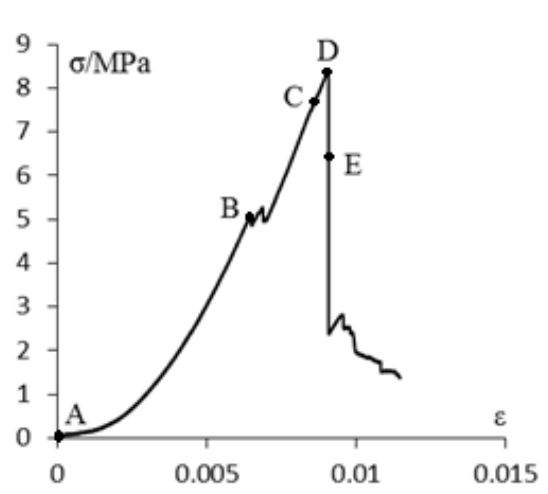

Fig.2 $\sigma-\varepsilon$ curve under $0 \mathrm{MPa}$ gas pressure

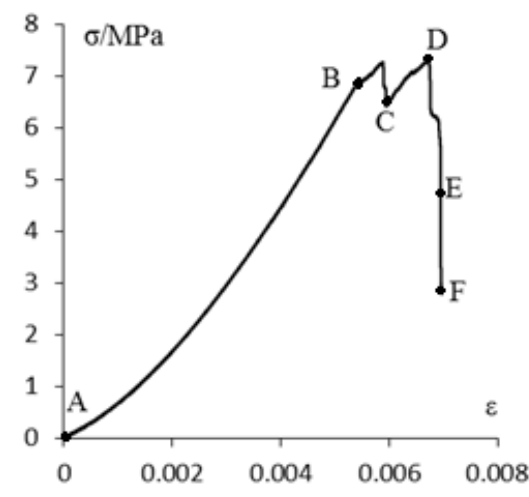

Fig.3 $\sigma-\varepsilon$ curve under 1MPa gas pressure

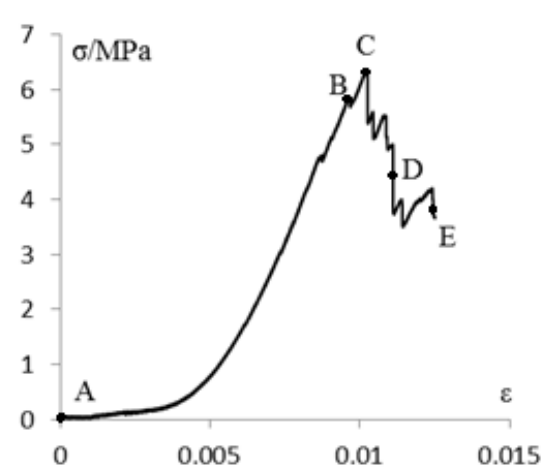

Fig.4 $\sigma-\varepsilon$ curve under $2 \mathrm{MPa}$ gas pressure 
Figure 2 and 5 are stress-strain curve and crack extension observation images of coal specimen conventional uniaxial compression experiment, and (a)-(e) in figure 5 corresponding to A-E in figure 2. Fig.5(a) is the image of specimen in the initial state $(\sigma=0)$, in which the surface of the specimen is complete with no obvious native fissure. With the increase of axial load, the first obvious crack in specimen surface appeared at yield point B $(\sigma=59.64 \% \sigma \mathrm{max})$, as shown in fig.5(b). Fig.5(c) is the photograph of coal sample before the peak stress $(\sigma=90.6 \% \sigma \mathrm{max})$, compared to the yield point the surface crack had no obvious change. Fig.5(d) is the observation images at peak point $(\sigma=\sigma \max )$, it can be seen severe cleft occurred in specimen surface and formed a main crack which bifurcated at the central of specimen, and induced several secondary cracks developed downward. Fig.5(e) is the observation image of the specimens after peak stress $\operatorname{point}(\sigma=73.4 \% \sigma \max )$, With the continual development of crack, the main crack penetrated and the width of crack increased at this stage, and the secondary cracks also developed apparently. Finally the main crack penetrated completely and specimen was disrupted, and the failure pattern is transverse tensile failure.

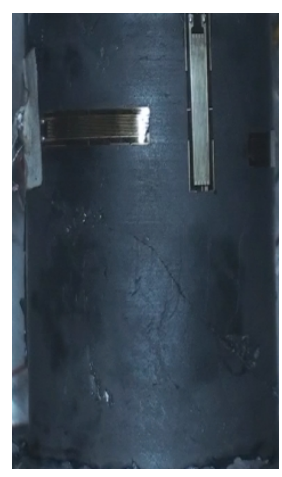

(a)

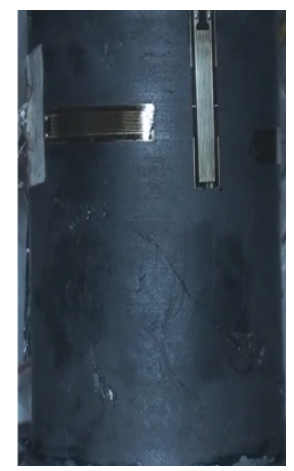

(b)

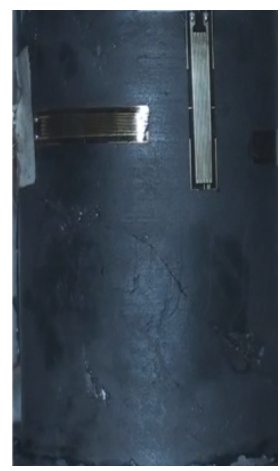

(c)

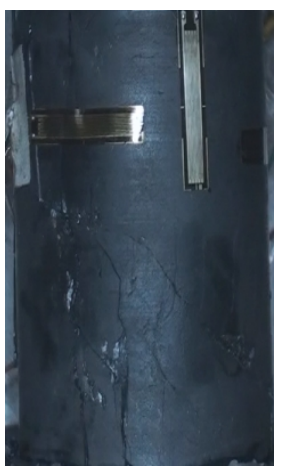

(d)

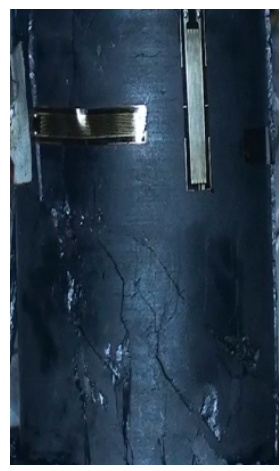

(e)

Fig.5 Crack development of coal under $0 \mathrm{MPa}$ gas pressure

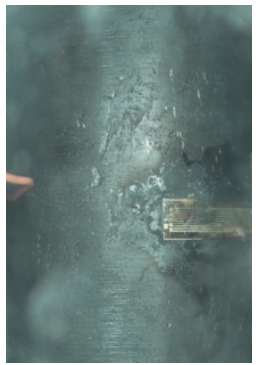

(a)

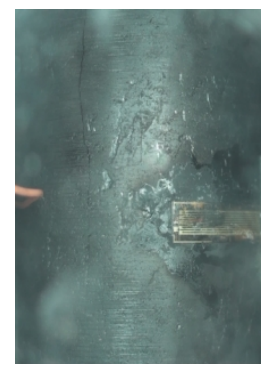

(b)

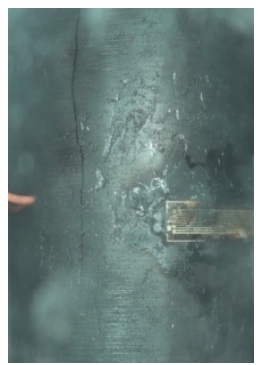

(c)

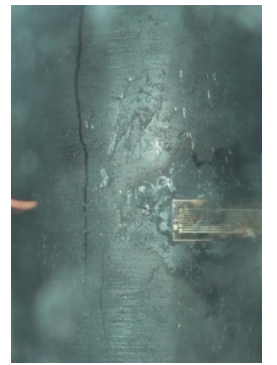

$\mathrm{S}(\mathrm{d})$

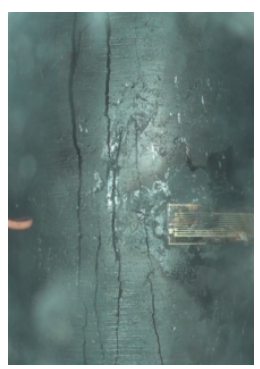

(e)

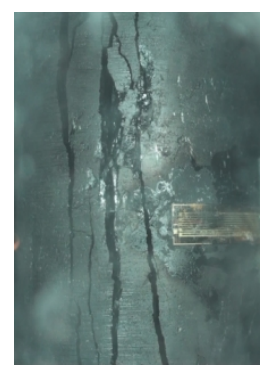

(f)

Fig.6 Crack development of coal under $1 \mathrm{MPa}$ gas pressure

Figure 3 and 6 are stress-strain curve and crack extension observation images of coal specimen in $1 \mathrm{MPa}$ gas pressure condition, and (a)-(f) in figure 6 corresponding to A-F in figure 3. Fig.6(a) is the image of specimen in the initial state $(\sigma=0)$, at this point the surface of coal sample is relatively complete and smooth, with no native cracks but only a small amount of tiny flaws. Fig.6(b) is image of coal sample surface at the yield point $(\sigma=93.5 \% \sigma \mathrm{max})$, After compaction stage and the elastic deformation stage, the native tiny flaws and fissures inside coal specimen has been compacted longitudinally, but no transverse stretching occurred; In yield stage, the first crack appeared upside the specimen, called 1\# crack. Fig.6(c) is image of specimen in middle of yield stage $(\sigma=90.3 \% \sigma \mathrm{max})$, with the increase of axial pressure , 1 \# crack extend downward with the increase of width, but it is not cut-through. Fig.6(d) is the picture of coal sample at peak stress point

$(\sigma=\sigma \max )$, at this point $1 \#$ crack continually extend and expand, and a new crack appeared on the 
lower right of specimen, called 2\# crack. Fig.6(e) is image of coal specimen after peak point ( $\sigma=60.4 \% \sigma \max$ ), 1\# crack is still expanding downward and nearly breakthrough; While 2\# crack not evolves obviously; And there were two new cracks appeared at left side of the specimen, called $3 \#$ and 4\# crack. Fig.6(f) is images of specimen when destroyed ( $\sigma=40.2 \% \sigma m a x)$, each crack has obviously expand and completely cut-through, the specimen is disrupted and the experiment ended. Failure pattern of coal specimen is transverse tensile failure.

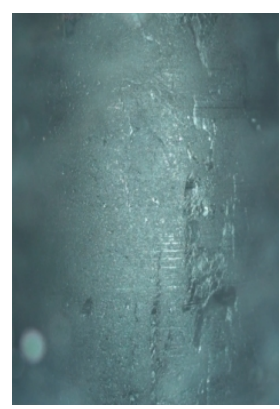

(a)

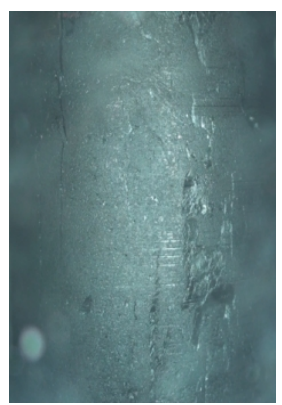

(b)

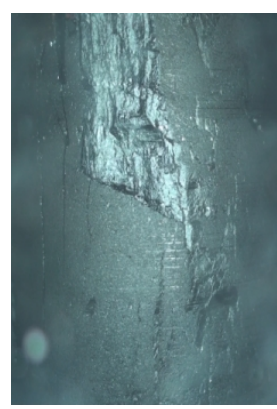

(c)

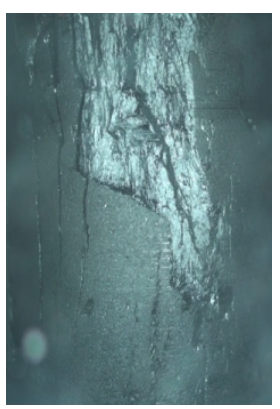

(d)

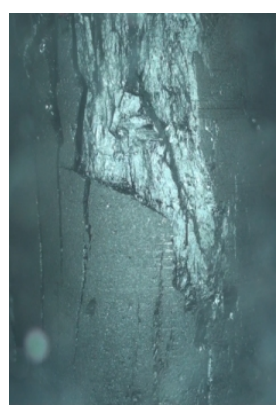

(e)

Fig.7 Crack development of coal under $2 \mathrm{MPa}$ gas pressure

Figure 4 and 7 are stress-strain curve and crack extension observation images of coal specimen in $2 \mathrm{MPa}$ gas pressure condition, and (a)-(e) in figure 7 corresponding to A-E in figure 4. Fig.7(a) is image of coal specimen in the initial state $(\sigma=0)$, and the surface of the specimen is complete with no obvious native fissure. Fig.7(b) is image of coal specimen in the yield stage $(\sigma=92.2 \% \sigma \mathrm{max})$, and the crack initiation occurred at upside of the specimen, called 1\# crack, Fig.7(c) is image of coal specimen at peak point $(\sigma=\sigma \max )$, and coal surface began to crumble, new cracks appeared at both sides of 1\#crack. After peak point as shown in Fig.7(d) $(\sigma=76.2 \% \sigma \mathrm{max})$, the width of all three cracks increased and cracks extended in parallel downward. Fig.7(e) is image of coal specimen before failure $(\sigma=56.7 \% \sigma \max )$, and the three cracks expanded rapidly with severe collapse at surface of specimen, after that the specimen is disrupted and the experiment ended. The failure pattern is also transverse tensile failure.

\section{Contrast of crack evolve progress}

Contrast and analyze the Crack extension progress of raw coal specimen in different gas pressure condition. Uniaxial compression failure process of raw coal could be divided into four stages $^{[15]}$ : (1)the micro-cracks compaction stage,(2)the micro-cracks compaction stage(3) the yield stage (from the yield point to the peak point), (4)post-peak stage (after the peak point), and come to the following conclusions:

(i)In initial state, all the specimen surface are complete with only a small amount of defects and no native crack exist.

(ii)In compaction stage and elastic stage, the specimens mainly perform longitudinal deformation, with no obvious crack in surface.

(iii) At yield stage, due to the increases of specimen deformation, the crack initiation and extension occurred and the initiation point is nearing yield point.

(iv) after the peak point, stress had a sharp decline process, and with the matching is the rapid development of specimen surface crack, embodied in: the extension of the main crack, increase of crack width, crack bifurcation, formation of new surface crack, etc.; 
(v) In post-peak stage, along with the rapid development of crack, the cutting-through of main crack all happened at this stage, then specimen was transverse tensed and eventually lead to the failure of the specimen

Comparative analysis of coal specimens peak stress under different gas pressure are shown in table 1. With the increase of gas pressure, peak stress of the specimen reduced. This is because the existence of gas pressure provides an expansion stress to the inner of coal and reduces the maximum pressure that coal specimen can withstand.

Table.1 Peak stress and crack initiation stress level of raw coal under different gas pressure

\begin{tabular}{cccc}
\hline$p / \mathrm{MPa}$ & $\sigma_{\max } / \mathrm{MPa}$ & $\sigma / \mathrm{MPa}$ & $\mathrm{K}=\sigma / \sigma_{\max }$ \\
\hline 0 & 8.40 & 5.01 & $59.64 \%$ \\
1 & 7.29 & 6.80 & $93.28 \%$ \\
2 & 6.36 & 5.92 & $93.08 \%$ \\
\hline
\end{tabular}

Comparative analysis of crack initiation stress level $\mathrm{K}$ of coal specimen under different gas pressure is shown in table 1. Define crack initiation stress level $\mathrm{K}$ as:

$$
K=\sigma / \sigma_{\max }
$$

Among them, $\sigma$ and $\sigma$ max represent crack initiation stress and peak stress of specimens under different gas pressure.

Analysis showed that: K-value under gas pressure is much bigger than K-value without gas pressure. This shows that the existence of gas pressure can delay the crack initiation of specimen; but the gas pressure size does not influence crack initiation stress level $\mathrm{K}$.

\section{Damage evolution in the failure process}

Coal is a kind of anisotropic porous material, with micro-defects and micro-cracks inside. The deformation and failure of coal is a complicated process of damage evolution, which is a irreversible change of the internal structure. In order to characterize the damage evolution regularity of coal, according to the strain equivalence principle ${ }^{[16][17]}$, the damage variable is defined based on the elastic modulus:

$$
D=1-\frac{E^{\prime}}{E}
$$

Among them, $D$ is the damage variable $(D=0$, the specimen was complete, while $D=1$, the specimen was completely disrupted.) $\mathrm{E}^{\prime}$ is the elastic modulus of damaged materials; $\mathrm{E}$ is the elastic modulus of the material.

$$
D=1-\frac{\sigma-\sigma_{0}}{E\left(\varepsilon-\varepsilon_{0}\right)}
$$

In the formula, $\mathrm{D}$ is the damage variable, $\sigma 0$ and $\varepsilon 0$ are the stress and Strain value of specimen at starting point of elastic stage; $\mathrm{E}$ is the elastic modulus. Thus the D- $\varepsilon$ Curve of damage evolution of the specimen (take the specimen under $1 \mathrm{MPa}$ gas pressure for example) was obtained. As shown in figure 8: 


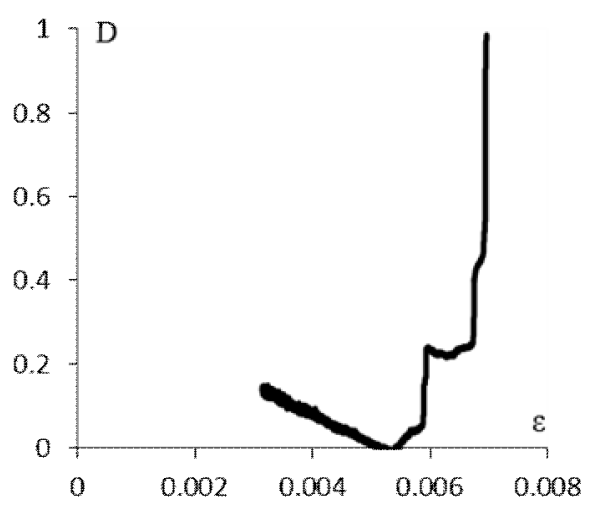

Fig.8 $D$ - $\varepsilon$ curcle of damage development

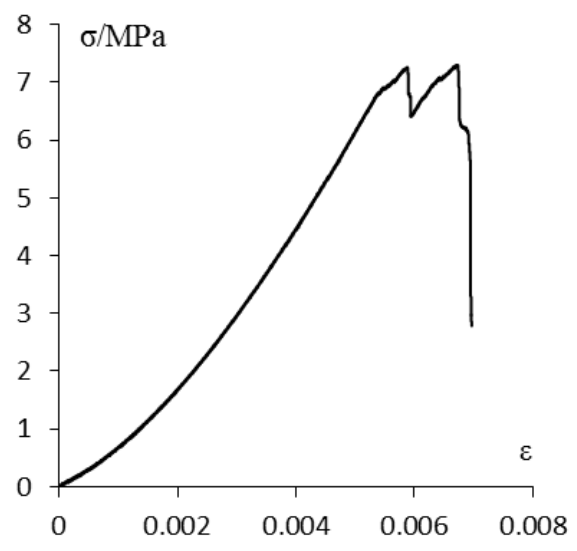

Fig.9 $\sigma-\varepsilon$ curcle of coal

For the convenience of Comparative analysis, the coal stress-strain curve was shown in figure 9. In the initial stage $(\sigma=0)$, the coal damage value is considered to be an initial porosity rate ${ }^{[18]}$ :

$$
D=\eta
$$

In compaction stage and elastic stage, with the micro-cracks in the coal longitudinally compacted and closed, with the porosity rate of the coal is reduced, and formed no transverse crack and damage. So the damage variable $\mathrm{D}$ at this stage is a slowly declining curve. In yield stage, inner crack began to evolve and surface crack appeared, $\sigma-\varepsilon$ curve slowed down while D- $\varepsilon$ curve began to arise. In post-peak stage, surface crack of coal developed rapidly, the main crack was cut-through and widen, Damage value D sharply increased, Corresponding to the rapidly reduce of $\sigma$ in $\sigma-\varepsilon$ curve after peak point, and Ultimately damage value $\mathrm{D}$ nearly to 1 at specimens failure.

\section{Summary}

Based on the uniaxial compression experiments of raw coal specimen under different gas pressure, observed and analyzed coal surface crack evolution in failure process Combined with macroscopical failure process, and studied the damage evolution law through failure process. Got the following conclusions:

(1)By the uniaxial compression experiment of raw coal under different gas pressure, got the regularity of surface crack evolution as follow:

In compaction stage and elastic stage, no crack initiation occurred at surface of coal; the crack initiation point appeared near the yield point; the cracks expanded and extended in yield stage; at peak point, the cracks vigorously evolved with the furcation of main crack, formation of secondary crack and increasing of crack width, etc.; in post-peak stage cracks evolved fast and main crack cut-through; and finally the specimen was disrupted.

(2)compare the influence of gas pressure to coal failure and concluded that, the higher the gas pressure, the smaller the peak stress of coal; the existence of gas pressure can delay the crack initiation of specimen, but the gas pressure size does not influence crack initiation stress level K.

(3) Defined the calculation method of damage variable D based on the change of elastic modulus, and found that the damage value of coal increased obviously from the yield point, and then increased sharply after peak point, finally at the time of failure, the damage value closed to 1. 


\section{References}

[1] BATZLE M L, SIMMONS G, SIEFRIED R W. Microcrack closure in rocks under stress: direct observation[J]. Jounal of Geophysical Research, 1980, 85: 7072-7090.

[2] ROBINA H C, WONG K T, CHAU P WANG. Microcracking and grain size effect in Yuen long marbles[J]. International Journal of Rock Mechanics and Mining Sciences, 1996, 33(5): 479-485.

[3] YOSSEF H HATZOR, ALON ZUR, YAAKOV MIMRAN. Microstructure effects on microcracking and brittle failure of dolomites[J]. Tectonophysics, 1997, 281: 141-161.

[4] SHANG Jialan,KONG Changjing,LI Tingjie,et al. Observation and study on meso-damage and fracture of rock[J]. Journal of Experimental Mechanics, 1999,14(3):373-383.

[5] NI Xiaohui, ZHU Dezhen, ZHAO Jie, et al. Meso-Damage Mechanical Digitalization Test of Complete Process of Rock Failure[J]. Rock and Soil Mechanics,2009,30 (11) :3283-3290.

[6] Kawakata H, Cho A, Yanagidani T, et al. The observations of faulting in Westerly granite under triaxial compression by X-ray CT scan[J]. Int J Rock Mech \& Min Sci, 1997, 34( 3-4) : 151-162.

[7] GE Xiurun, REN Jianxi,PU Yibin,et al. Real-time CT test on the meso-damage development law of rock[J]. Science in China:Series E,2000,30(2):104-111.

[8] DAI Gaofei,YIN Guangzhi,PI Wenli. Experimental Study on Meso-Damage Evolution of Coal under Uniaxial Compression[J]. Journal of Tongji University:Natural Science, 2004,32(5):591-595.

[9]Liu Dongmei, Xie Heping, Zhou Yubin, et al. Real-Time Testing Study of Rock Fracture under Coupled Compression and Shear[J]. Chinese Journal of Rock Mechanics and Engineering,2004,23(10):1616-1620.

[10] Liu Dongmei, Cai Meifeng, Zhou Yubing, et al. Dynamic Monitoring on Developing Process of Rock Cracks[J]. Chinese Journal of Rock Mechanics and Engineering,2006,25(3):467-472.

[11] XU Jiang,TAN Haoyue,WANG Lei,et al. Meso-Evolution Process Of Gas-Containing Coal Shear Failure Under Different Normal Stresses[J]. Chinese Journal of Rock Mechanics and Engineering,2012,31(6):1192-1197.

[12] Xu Jiang, Lu Lifeng, Yang Hongwei, et al. Study of Evolution Law of Microfracturing Progress of Sandstone under Shear Loading[J]. Chinese Journal of Rock Mechanics and Engineering,2011,30 (5):944-950.

[13] Xu Jang,Liu Jing, Cheng Lichao, et al. Mesoscopic Cracking and Expansion of Double Sheared Sandstone under Compressive-Shear Stress[J]. Chinese Journal of Rock Mechanics and Engineering,2014,33(4) :649-657.

[14] Xu Jang,PENG Shoujian, YIN Gangzhi,et al. Development of meso-shear test equipment for coal rock containing gas and its application[J].Chinese Journal of Rock Mechanics and Engineering,2011,30(4):677-685.

[15]Cai Meifeng, He Manchao, Liu Dongyan, et al. Rock Mechanics and Engineering [M]. Beijing:Science Press, 2004.

[16] YU Shouwen, FENG Xiqiao. Damage mechanics [M]. Bei Jing: Tsing Hua University Press.1997.

[17] LI He, YIN Guangzhi, XU Jiang, et al. Rock Fracture Mechanics[M].Chong Qing: Chong Qing University Press, 1988

[18] GUO Zihong, JU Yang, DONG Yuli, et al. Experiment Study Of Rock Damage Deformation Characteristics Based On Plastic Volumetric Strain Analysis[J].Journal Of Experimental Mechanics, 2010,25(3):293-298. 